minimal residual disease in patients with core binding factor acute myeloid leukemia. Blood. 2013;121:2213-23.

4. Yin JA, O'Brien MA, Hills RK, Daly SB, Wheatley K, Burnett AK. Minimal residual disease monitoring by quantitative RT-PCR in core binding factor AML allows risk stratification and predicts relapse: results of the United Kingdom MRC AML-15 trial. Blood. 2012;120:2826-35.

5. Weisser M, Haferlach C, Hiddemann W, Schnittger S. The quality of molecular response to chemotherapy is predictive for the outcome of AML1-ETO-positive AML and is independent of pretreatment risk factors. Leukemia. 2007;21:1177-82.

6. Faber ZJ, Chen X, Gedman AL, Boggs K, Cheng J, Ma J, et al. The genomic landscape of core-binding factor acute myeloid leukemias. Nat Genet. 2016;48:1551-6.

7. Duployez N, Marceau-Renaut A, Boissel N, Petit A, Bucci M, Geffroy S, et al. Comprehensive mutational profiling of core binding factor acute myeloid leukemia. Blood. 2016;127:2451-9.

8. Nucifora G, Larson RA, Rowley JD. Persistence of the 8;21 translocation in patients with acute myeloid leukemia type M2 in long-term remission. Blood. 1993;82:712-5.

9. Pigazzi M, Manara E, Buldini B, Beqiri V, Bisio V, Tregnago C, et al. Minimal residual disease monitored after induction therapy by RQ-PCR can contribute to tailor treatment of patients with $t$ $(8 ; 21)$ RUNX1-RUNX1T1 rearrangement. Haematologica. 2015;100:e99-101.
10. Allen C, Hills RK, Lamb K, Evans C, Tinsley S, Sellar R, et al. The importance of relative mutant level for evaluating impact on outcome of KIT, FLT3 and CBL mutations in core-binding factor acute myeloid leukemia. Leukemia. 2013;27:1891-901.

11. Jahn N, Agrawal M, Bullinger L, Weber D, Corbacioglu A, Gaidzik VI, et al. Incidence and prognostic impact of ASXL2 mutations in adult acute myeloid leukemia patients with $\mathrm{t}(8 ; 21)$ (q22; q22): a study of the German-Austrian AML Study Group. Leukemia. 2017;31:1012-5.

12. Yamato G, Shiba N, Yoshida K, Shiraishi Y, Hara Y, Ohki K, et al. ASXL2 mutations are frequently found in pediatric AML patients with $\mathrm{t}(8 ; 21) / \mathrm{RUNX1-RUNX1T1}$ and associated with a better prognosis. Genes Chromosomes Cancer. 2017;56:382-93.

13. Micol JB, Duployez N, Boissel N, Petit A, Geffroy S, Nibourel O, et al. Frequent ASXL2 mutations in acute myeloid leukemia patients with $\mathrm{t}(8 ; 21) / \mathrm{RUNX1-RUNX1T1}$ chromosomal translocations. Blood. 2014;124:1445-9.

14. Stone RM, Mandrekar SJ, Sanford BL, Laumann K, Geyer S, Bloomfield CD, et al. Midostaurin plus Chemotherapy for Acute Myeloid Leukemia with a FLT3 Mutation. N Engl J Med. 2017;377:454-64.

15. Evans EK, Gardino AK, Kim JL, Hodous BL, Shutes A, Davis A, et al. A precision therapy against cancers driven by KIT/PDGFRA mutations. Sci Transl Med. 2017;2017:9.

\title{
U2AF1 mutation types in primary myelofibrosis: phenotypic and prognostic distinctions
}

\author{
Ayalew Tefferi ${ }^{1} \cdot$ Christy M. Finke ${ }^{1} \cdot$ Terra L. Lasho $^{1} \cdot$ Curtis A. Hanson $^{2} \cdot$ Rhett P. Ketterling $^{3} \cdot$ Naseema $_{\text {Gangat }}{ }^{1}$. \\ Animesh Pardanani ${ }^{1}$
}

Received: 2 December 2017 / Revised: 21 January 2018 / Accepted: 26 January 2018 / Published online: 27 February 2018

(c) The Author(s) 2018. This article is published with open access

It is now well established that approximately 90\% of patients with primary myelofibrosis (PMF) express one of

Electronic supplementary material The online version of this article (https://doi.org/10.1038/s41375-018-0078-0) contains supplementary material, which is available to authorized users.

Ayalew Tefferi

tefferi.ayalew@mayo.edu

1 Division of Hematology, Departments of Medicine and Laboratory Medicine, Mayo Clinic, Rochester, MN, USA

2 Division of Hematopathology, Departments of Medicine and Laboratory Medicine, Mayo Clinic, Rochester, MN, USA

3 Division of Cytogenetics, Departments of Medicine and Laboratory Medicine, Mayo Clinic, Rochester, MN, USA three "driver" mutations $(J A K 2, C A L R$, and $M P L)$ that are often mutually exclusive and shown to be phenotypically and prognostically relevant [1]. In addition, targeted sequencing has revealed other mutations or DNA variants in more than $80 \%$ of patients with PMF, the most frequent being ASXL1 (36\%), TET2 (18\%), SRSF2 (18\%), and U2AF1 (16\%) [2]. ASXL1 and SRSF2 mutations have consistently been shown to be prognostically detrimental in PMF [1], while other mutations, such as $S F 3 B 1$ and $U 2 A F 1$, were phenotypically characterized by ring sideroblasts [3] and anemia/thrombocytopenia [4], respectively. The unique association between $U 2 A F 1$ mutations and anemia and/or thrombocytopenia has also been demonstrated in myelodysplastic syndromes (MDS) [5]. In both PMF and MDS, $U 2 A F 1$ mutations were associated with inferior survival, which, however, might have been accounted for by their 
association with other previously recognized risk factors, including anemia and thrombocytopenia $[4,6,7]$. In the current study, we classified $U 2 A F 1$ mutations in PMF into the two main mutation variants, Q157 and S34, in order to examine differences in phenotype and prognostic relevance. The rationale for grouping $U 2 A F 1$ mutations into these two mutation variants include (i) almost all PMF patients harbor one of these two mutations, (ii) classification in the two specific $U 2 A F 1$ subtypes has already been assigned in MDS, and (iii) there is laboratory information that suggests functional differences between U2AF1-splice site interactions for S34 vs. Q157 variants [8, 9].

The current study was approved by the institutional review board (IRB). Diagnoses and treatment approaches were in accordance with what was considered standard of care at the time of initial diagnosis or first referral [10]. Samples for mutation analysis included archived DNA from bone marrow or peripheral blood collected at time of patient referral, under IRB-approved protocols. Prognostically relevant mutations were screened by previously described methods, which included target-capture next generation sequencing [2] or standard PCR techniques and bidirectional sequencing [11]; specifically for $U 2 A F 1$, we amplified two areas with known mutations that included residues S34 and Q157. Briefly, two separate PCR reactions were performed and the primers used were as follows: (for S34) Forward: 5'-GGTGCTTAATACCACGGAAAA-3'; Reverse: 5'-AGTCGATCACCTGCCTCACT-3'; (for Q157) Forward: 5'-GCCTCGTGTGCATTCTCTG-3'; Reverse: 5'-CTTTTC AGTTTCGCCGTGAG-3'. PCR amplification conditions were the same for both $U 2 A F 1$ reactions and included a primary denaturation of $95^{\circ} \mathrm{C}$ for $2 \mathrm{~min}$, followed by 35 cycles of: $95^{\circ} \mathrm{C}$ for $30 \mathrm{~s}, 57^{\circ} \mathrm{C}$ for $45 \mathrm{~s}$, and $72^{\circ} \mathrm{C}$ for 45 $\mathrm{s}$; and ended with a final extension of $72{ }^{\circ} \mathrm{C}$ for $5 \mathrm{~min}$. Products were purified before sequencing. Statistical analyses considered clinical and laboratory parameters obtained at time of diagnosis or first referral, which coincided, in all instances, with time of sample collection for mutation analysis. Conventional methods were used for statistical analysis (JMP ${ }^{\circ}$ Pro 13.0.0 software; SAS Institute, Cary, NC). Survival analysis was computed from the date of diagnosis or first referral to date of event. Patients receiving allogeneic stem cell transplant were censored at the time of their transplantation. Date of leukemic transformation replaced date of death, as the uncensored variable, for estimating leukemia-free survival. Cox proportional hazard regression model was used for multivariable analysis.

A total of 491 patients (median age 64 years; $62 \%$ males) were annotated for $U 2 A F 1$ mutations. Driver mutation distributions were $60 \%$ JAK2, $19 \%$ type $1 /$ like $C A L R, 4 \%$ type $2 /$ like $C A L R, 7 \% M P L$ and $10 \%$ triple negative. Dynamic international prognostic scoring system (DIPSS) distributions were $10 \%$ high, 38\% intermediate-2, 36\% intermediate-1 and 16\% low [12]. Spleen was palpable in $75 \%$ of patients and measured $>10 \mathrm{~cm}$ below the left costal margin in $33 \%$. Cytogenetic risk categories, according to the recently revised system[13], were very high risk in 7\%, unfavorable in $16 \%$ and favorable in $78 \%$. Mutational frequencies were $16 \%$ for $U 2 A F 1,38 \%$ for $A S X L 1,14 \%$ for $S R S F 2,4 \%$ for $E Z H 2$ and $5 \%$ for $I D H 1 / 2$. In order to assist with detailed examination of the previously recognized relationship between $U 2 A F 1$ mutations and cytopenia, four separate categories of anemia and three of thrombocytopenia were considered; anemia was absent in $17 \%$ of patients while it was classified as being severe (transfusion dependent or $<8 \mathrm{~g} / \mathrm{dl}$ in women or $<9 \mathrm{~g} / \mathrm{dl}$ in men) in $33 \%$, moderate (hemoglobin $<10 \mathrm{~g} / \mathrm{dl}$ in women and $<11 \mathrm{~g} / \mathrm{dl}$ in men) in $20 \%$ and mild (hemoglobin below the sex-adjusted lower limit of normal) in 30\%; reference ranges for hemoglobin, at our institution, were $13.5-17.5 \mathrm{~g} / \mathrm{dl}$ for men and $12.0-15.5 \mathrm{~g} / \mathrm{dl}$ for women. Platelet count was $450 \times 10(9) / 1$ or more in $18 \%$ of patients, between 100 and $450 \times 10(9) / 1$ in $60 \%$ and below $100 \times 10(9) / 1$ in $22 \%$.

Among the 77 (16\%) patients with $U 2 A F 1$ mutations, 50 $(65 \%)$ involved Q157 \pm other concomitant variants, 26 (34\%) involved S34 \pm other concomitant variants not including Q157 and one involved neither (see Fig. 1 for details). For the purposes of comparing the phenotypic and prognostic differences between distinct $U 2 A F 1$ mutational types, we grouped all Q157 variants together, regardless of the presence of concomitant other U2AF1 mutations. Accordingly, comparison of the three major $U 2 A F 1$ mutational categories (414 unmutated vs. 50 mutated for Q157 \pm other concomitant variants vs. 26 mutated for S34 \pm other concomitant variants not including Q157) disclosed the following significant associations: both Q157 and S34 variants with anemia (Fig. $1 ; p<0.01$ ), JAK2 and $M P L$ mutations (Fig. $1 ; p<0.01$ ) and absence of marked splenomegaly defined by spleen palpable at $>10 \mathrm{~cm}$ below the costal margin $(p=0.02)$. In addition, Q157, but not S34, was associated with thrombocytopenia (Fig. 1; $p=0.02$ ), older age $(p<0.01), A S X L 1$ mutations $(p<0.01 ;$ Fig. 1$)$ and constitutional symptoms $(p<0.01)$. Multiple logistic regression analysis confirmed the association between Q157 mutation and anemia, thrombocytopenia, driver mutational status, ASXL1 mutations and absence of marked splenomegaly; significance was lost for age and constitutional symptoms (data not shown). Significant associations were not evident for cytogenetic risk categories $(p=0.93)$, circulating blast percentage $(p=0.47)$, leukocyte count $(p=$ $0.6)$, or $E Z H 2(p=0.28)$ or $I D H 1 / 2$ mutations $(p=0.44)$.

After a median follow-up of 3.9 years, $338(69 \%)$ deaths, $51(10 \%)$ leukemic transformations and $33(7 \%)$ stem cell transplants were recorded. Overall survival was significantly shorter for patients with $U 2 A F 1$ Q157 mutations, 

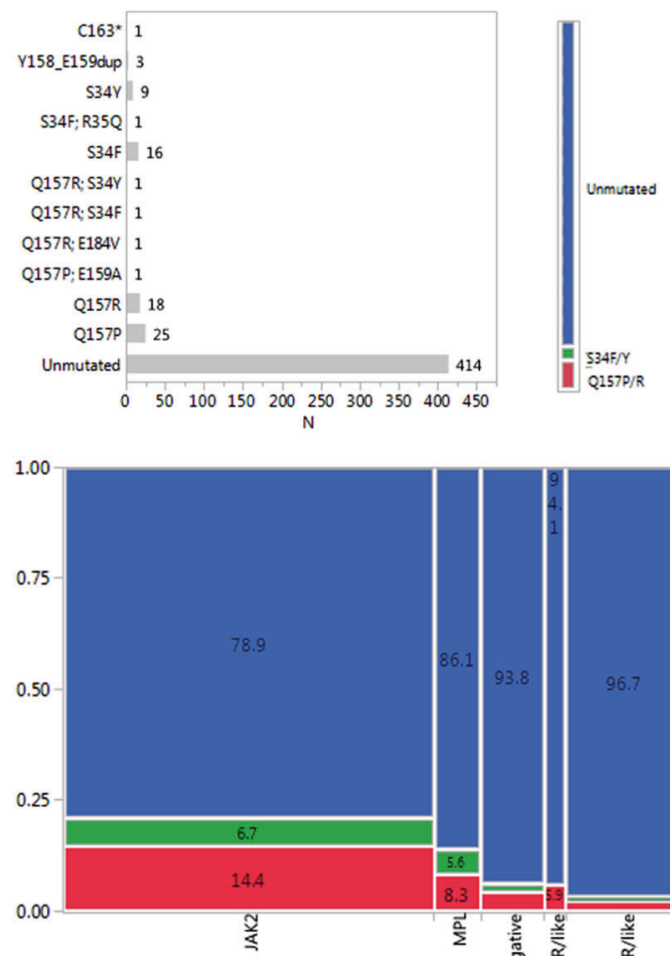

Driver mutations
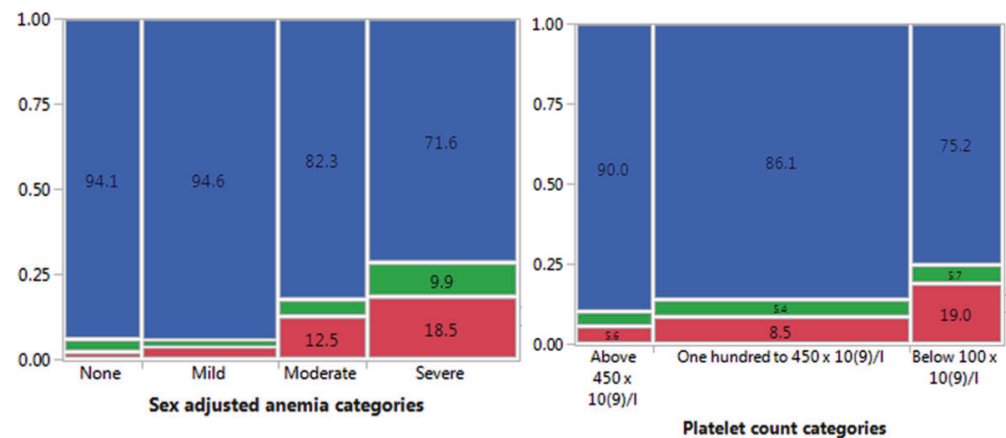

Platelet count categories

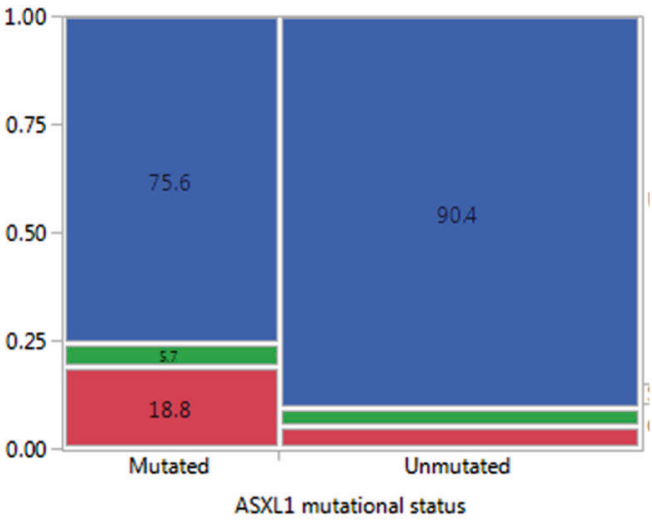

Fig. 1 U2AF1 mutation types among 491 patients with primary myelofibrosis and comparison of $U 2 A F 1$ Q157 vs. U2AF1 S34 vs. U2AF1 unmutated categories, in regards to anemia, thrombocytopenia, driver mutational status, and ASXL1 mutations
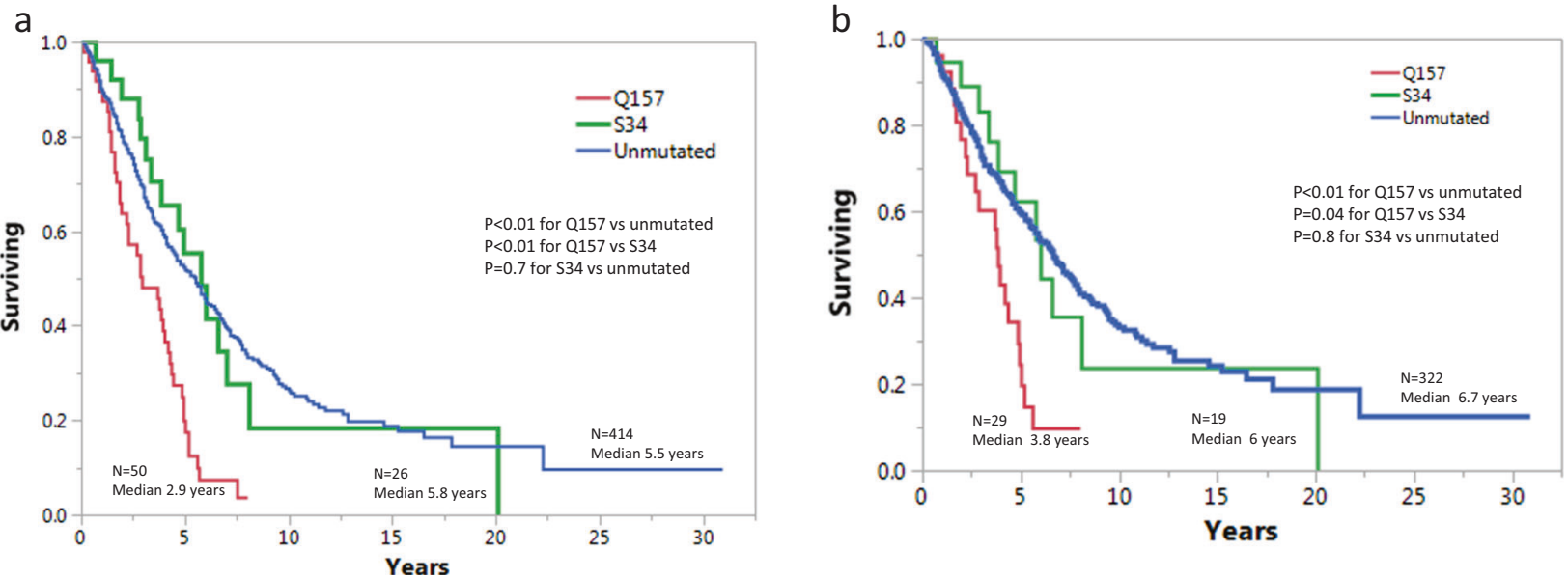

Fig. 2 a Survival data on 490 patients with primary myelofibrosis stratified by U2AF1 mutation types. b Survival data on 370 transplant-age patients with primary myelofibrosis (age 70 years or younger), stratified by U2AF1 mutation types

compared to those with $U 2 A F 1 \mathrm{~S} 34$ mutations or $U 2 A F 1$ unmutated cases (Fig. 2a, b); the survival effect was most evident in younger patients, where the information is important for making treatment decisions (Fig. 2b). The results were unchanged when analysis was limited to patients who harbored only Q157 variant $(n=43)$, only S34 variant $(n=25)$ or were unmutated for any $U 2 A F 1$ mutation $(n=414)$; HRs $(95 \% \mathrm{CI})$ were $2.3(1.6-3.2)$ for Q157 vs. U2AF1 unmutated, $2.1(1.2-4.0)$ for Q157 vs. S34 and $1.1(0.6-1.7)$ for $\mathrm{S} 34$ vs. $U 2 A F 1$ unmutated. The prognostic relevance of $U 2 A F 1$ Q157 mutations was not accounted for by age $(p=0.01)$, anemia $(p=0.007)$, 
thrombocytopenia $(p<0.001)$, driver mutational status $(p<0.001)$ or $A S X L 1$ mutations $(p<0.001)$. Multivariable analysis confirmed the independent prognostic contribution of $U 2 A F 1$ Q157 but not S34 mutation type, both in the context of genetic risk factors and DIPSS (Supplemental Table 1). U2AF1 mutational status did not affect leukemiafree survival $(p=0.52)$. The overall results in terms of both phenotypic and prognostic differences were not affected by the removal of patients with dual mutations or those with Y158_E159dup.

$S F 3 B 1, S R S F 2$, and $U 2 A F 1$ constitute the three major spliceosome genes mutated in myeloid malignancies with the most frequent mutations occurring, respectively, in the K700, P95, and Q157/S34 hotspot codons [14]. These mutations are usually heterozygous and mutually exclusive and their mechanism of action involves altered $3^{\prime}$ splice site recognition. In myeloid neoplasms, U2AF1 mutations might contribute to impaired erythroid differentiation and lineage-specific alteration of mRNA splicing transcripts [15]. Considering differences between U2AF1splice site interactions for S34 vs. Q157 variants [8, 9], it is important to examine the clinical implications of different mutation types. The majority of $U 2 A F 1$ mutations in MDS affect the S34 codon (approximately 72 vs. $30 \%$ for Q157 mutation type, in one study) [7], which is opposite of the pattern seen in the current study for PMF patients (65\% for Q157 vs. 34\% for S34) [5]. The observations in MDS and PMF are also different in regards to the age distribution of $U 2 A F 1$ mutated patients; younger in MDS and older in PMF [5-7]. Similarly, we did not find $U 2 A F 1$ mutation associations with +8 or $20 \mathrm{q}$ - cytogenetic abnormalities, as had been previously reported in MDS (data not shown) $[5,7]$. On the other hand, in both MDS and PMF, U2AF1 mutations were associated with anemia, thrombocytopenia, and inferior survival [6, 7]. In MDS, thrombocytopenia was specifically associated with the S34 and anemia with the Q157 U2AF1 mutation types [5]. By contrast, in the current study with PMF patients, both mutation types were associated with anemia and the association with thrombocytopenia was most evident for the Q157 mutation type. Regardless, the current study establishes $U 2 A F 1$ Q157, but not S34, as a candidate mutation for inclusion in future genetics-based risk stratification models; in this regard, it is noteworthy to recall our previously reported observations with ASXL1 mutations, where mutation type did not appear to matter [16]. Our observations in the current study also underscore the need to stratify $U 2 A F 1$ mutated patients according to mutation types, especially in clinical trials involving splice modulating agents [17].

Author contributions: All authors have reviewed and approved the manuscript. C.A.H. reviewed pathology data. R.P.K. reviewed cytogenetic data. N.G. and A.P. contributed patients and assisted in study design and concept. T.L.L. and C.M.F. were in charge of molecular studies and analysis. A.T. developed the study concept and design, contributed patients, assisted in data extraction, performed statistical analysis and wrote the paper.

\section{Compliance with ethical standards}

Conflict of interest The authors declare that they have no conflict of interest.

Open Access This article is licensed under a Creative Commons Attribution-NonCommercial-NoDerivatives 4.0 International License, which permits any non-commercial use, sharing, distribution and reproduction in any medium or format, as long as you give appropriate credit to the original author(s) and the source, and provide a link to the Creative Commons license. You do not have permission under this license to share adapted material derived from this article or parts of it. The images or other third party material in this article are included in the article's Creative Commons license, unless indicated otherwise in a credit line to the material. If material is not included in the article's Creative Commons license and your intended use is not permitted by statutory regulation or exceeds the permitted use, you will need to obtain permission directly from the copyright holder. To view a copy of this license, http://creativecommons.org/licenses/by-nc-nd/4.0/.

\section{References}

1. Vannucchi AM, Lasho TL, Guglielmelli P, Biamonte F, Pardanani A, Pereira A, et al. Mutations and prognosis in primary myelofibrosis. Leukemia. 2013;27:1861-9.

2. Tefferi A, Lasho TL, Finke CM, Elala Y, Hanson CA, Ketterling $\mathrm{RP}$, et al. Targeted deep sequencing in primary myelofibrosis. Blood Adv. 2016;1:105-11.

3. Lasho TL, Finke CM, Hanson CA, Jimma T, Knudson RA, Ketterling RP, et al. SF3B1 mutations in primary myelofibrosis: clinical, histopathology and genetic correlates among 155 patients. Leukemia. 2012;26:1135-7.

4. Barraco D, Elala YC, Lasho TL, Begna KH, Gangat N, Finke C, et al. Molecular correlates of anemia in primary myelofibrosis: a significant and independent association with U2AF1 mutations. Blood Cancer J. 2016;6:e415.

5. Li B, Liu J, Jia Y, Wang J, Xu Z, Qin T, et al. Clinical features and biological implications of different U2AF1 mutation types in myelodysplastic syndromes. Genes Chromosomes Cancer. 2018;57:80-88. https://doi.org/10.1002/gcc.22510. Epub 2017 Nov 23

6. Tefferi A, Finke CM, Lasho TL, Wassie EA, Knudson R, Ketterling RP, et al. U2AF1 mutations in primary myelofibrosis are strongly associated with anemia and thrombocytopenia despite clustering with JAK2V617F and normal karyotype. Leukemia. 2014;28:431-3.

7. Wu SJ, Tang JL, Lin CT, Kuo YY, Li LY, Tseng MH, et al. Clinical implications of U2AF1 mutation in patients with myelodysplastic syndrome and its stability during disease progression. Am J Hematol. 2013;88:E277-282.

8. Kielkopf CL. Insights from structures of cancer-relevant pre-mRNA splicing factors. Curr Opin Genet Dev. 2017;48: $57-66$.

9. Ilagan JO, Ramakrishnan A, Hayes B, Murphy ME, Zebari AS, Bradley $\mathrm{P}$, et al. U2AF1 mutations alter splice site recognition in hematological malignancies. Genome Res. 2015;25:14-26.

10. Vardiman JW, Thiele J, Arber DA, Brunning RD, Borowitz MJ, Porwit A, et al. The2008 revision of the World Health 
Organization (WHO) classification of myeloid neoplasms and acute leukemia: rationale and important changes. Blood. 2009;114:937-51.

11. Patnaik MM, Lasho TL, Finke CM, Hanson CA, Hodnefield JM, Knudson RA, et al. Spliceosome mutations involving SRSF2, SF3B1, and U2AF35 in chronic myelomonocytic leukemia: prevalence, clinical correlates, and prognostic relevance. Am J Hematol. 2013;88:201-6.

12. Passamonti F, Cervantes F, Vannucchi AM, Morra E, Rumi E, Pereira A, et al. A dynamic prognostic model to predict survival in primary myelofibrosis: a study by the IWG-MRT (International Working Group for Myeloproliferative Neoplasms Research and Treatment). Blood. 2010;115:1703-8.

13. Leukemia. 2018 Feb 2. https://doi.org/10.1038/s41375-018-0018-z. [Epub ahead of print] Revised cytogenetic risk stratification in primary myelofibrosis: analysis based on 1002 informative patients. Tefferi A1, Nicolosi M2, Mudireddy M2, Lasho TL2, Gangat N2, Begna KH2, Hanson CA3, Ketterling RP4, Pardanani A2.

14. Inoue D, Bradley RK, Abdel-Wahab O. Spliceosomal gene mutations in myelodysplasia: molecular links to clonal abnormalities of hematopoiesis. Genes Dev. 2016;30:989-1001.

15. Yip BH, Steeples V, Repapi E, Armstrong RN, Llorian M, Roy S, et al. The U2AF1S34F mutation induces lineage-specific splicing alterations in myelodysplastic syndromes. J Clin Invest. 2017; 127:2206-21.

16. Leukemia. 2017 Nov 21. https://doi.org/10.1038/leu.2017.318. [Epub ahead of print] Prognostic significance of ASXL1 mutation types and allele burden in myelofibrosis. Tefferi A1, Lasho TL1, Finke C1, Gangat N1, Hanson CA2, Ketterling RP3, Pardanani A1

17. Lee SC, Abdel-Wahab O. Therapeutic targeting of splicing in cancer. Nat Med. 2016;22:976-86.

Leukemia (2018) 32:2278-2281

https://doi.org/10.1038/s41375-018-0059-3

Chronic lymphocytic leukemia

\title{
Sustained long-lasting responses after lenalidomide discontinuation in patients with chronic lymphocytic leukemia
}

\author{
Paolo Strati ${ }^{1}$ - Alessandra Ferrajoli ${ }^{1}$ - William G. Wierda ${ }^{1}$ Nitin Jain ${ }^{1} \cdot$ Philip A. Thompson $\mathbb{1 0}^{1} \cdot$ Susan M. O'Brien ${ }^{1}$. \\ Katy Rezvani ${ }^{1} \cdot$ Hagop M. Kantarjian ${ }^{1} \cdot$ Jan A. Burger ${ }^{1} \cdot$ Christina O. Hinojosa $^{1} \cdot$ Michael J. Keating ${ }^{1} \cdot$ Zeev Estrov $^{1}$
}

Received: 7 November 2017 / Revised: 11 January 2018 / Accepted: 17 January 2018 / Published online: 20 February 2018

(c) Macmillan Publishers Limited, part of Springer Nature 2018

Toxicity prompting treatment discontinuation remains a major challenge in the management of chronic lymphocytic leukemia (CLL), both with chemoimmunotherapy and with novel biological agents [1-3]. Toxicity-related treatment discontinuation was reported in up to $60 \%$ of CLL patients treated with the combination of fludarabine, cyclophosphamide, and rituximab [4, 5]. In patients treated with chemoimmunotherapy, treatment discontinuation can result in lower response rates and shorter time to next treatment (TTNT) [6]. Toxicity-related treatment discontinuation has been reported in about 10 to $20 \%$ of CLL patients treated with ibrutinib [7] and up to $40 \%$ of those treated with idelalisib plus rituximab [8]; typically, following discontinuation, the disease rapidly progresses with aggressive biological and clinical features and an overall unfavorable outcome $[7,8]$.

Zeev Estrov

zestrov@mdanderson.org

1 Department of Leukemia, The University of Texas MD Anderson Cancer Center, Houston, TX, USA
Lenalidomide is an immunomodulatory agent with wellestablished clinical benefits in CLL [9]. Unfortunately, its development has been slowed by the results of a phase III front-line clinical trial comparing single-agent lenalidomide with single-agent chlorambucil in elderly patients with CLL, which was stopped early because of higher rate of toxicity-related deaths in the lenalidomide arm [10].

Because lenalidomide affects the interaction between CLL cells and their immune microenvironment [11], we hypothesized that the clinical effects of lenalidomide persist after treatment discontinuation.

Two hundred eight patients with CLL received lenalidomide-based therapy on clinical trials at The University of Texas MD Anderson Cancer Center between December 2005 and October 2015. One hundred twenty patients received lenalidomide as front-line treatment and 88 as salvage therapy. Lenalidomide was given as a single agent in 75 patients and in combination with an anti-CD20 monoclonal antibody (rituximab or ofatumumab) in 123. Of these 208 patients, $43(21 \%)$ had treatment discontinued because of toxicity. To test our hypothesis, we analyzed the long-term outcomes of these patients. This study has been 